\title{
Particle flow rate from the airways as fingerprint diagnostics in mechanical ventilation in the intensive care unit: a randomised controlled study
}

\author{
Filip Hallgren ${ }^{1}$, Martin Stenlo ${ }^{2,3,4,5}$, Anna Niroomand ${ }^{3,4,5,6}$, Ellen Broberg ${ }^{2,3,4,5}$, Snejana Hyllén ${ }^{2,3,4,5}$, \\ Malin Malmsjö ${ }^{4}$ and Sandra Lindstedt $\mathbb{D}^{1,3,4,5}$
}

${ }^{1}$ Dept of Cardiothoracic Surgery and Transplantation, Skåne University Hospital, Lund University, Lund, Sweden. ${ }^{2}$ Cardiothoracic Anaesthesia and Intensive Care, Skåne University Hospital, Lund University, Lund, Sweden. ${ }^{3}$ Wallenberg Center for Molecular Medicine, Lund University, Lund, Sweden. ${ }^{4}$ Dept of Clinical Sciences, Lund University, Lund, Sweden. ${ }^{5}$ Lund Stem Cell Center, Lund University, Lund, Sweden. ${ }^{6}$ Rutgers Robert Wood Johnson Medical School, New Brunswick, NJ, USA.

Corresponding author: Sandra Lindstedt (sandra.lindstedt_ingemansson@med.lu.se)

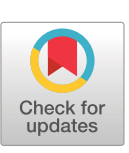

This version is distributed under the terms of the Creative Commons Attribution Non-Commercial Licence 4.0. For commercial reproduction rights and permissions contact permissions@ersnet.org

Received: 21 Dec 2020 Accepted: 23 May 2021

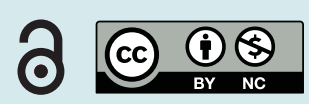

\section{Shareable abstract (@ERSpublications)}

Particle flow rate (PFR) from the airways may be used to continuously monitor the small airways in real time. A low PFR during mechanical controlled ventilation is likely to correspond to a protective ventilation. https://bit.ly/2RSklqL

Cite this article as: Hallgren F, Stenlo M, Niroomand A, et al. Particle flow rate from the airways as fingerprint diagnostics in mechanical ventilation in the intensive care unit: a randomised controlled study. ERJ Open Res 2021; 7: 00961-2020 [DOI: 10.1183/23120541.00961-2020].

\section{Abstract}

Introduction Mechanical ventilation can be monitored by analysing particles in exhaled air as measured by particle flow rate (PFR). This could be a potential method of detecting ventilator-induced lung injury (VILI) before changes in conventional parameters can be detected. The aim of this study was to investigate PFR during different ventilation modes in patients without lung pathology.

Method A prospective study was conducted on patients on mechanical ventilation in the cardiothoracic intensive care unit (ICU). A PExA 2.0 device was connected to the expiratory limb on the ventilator for continuous measurement of PFR in 30 patients randomised to either volume-controlled ventilation (VCV) or pressure-controlled ventilation (PCV) for 30 min including a recruitment manoeuvre. PFR measurements were continued as the patients were transitioned to pressure-regulated volume control (PRVC) and then pressure support ventilation (PSV) until extubation.

Results PRVC resulted in significantly lower PFR, while those on PSV had the highest PFR. The distribution of particles differed significantly between the different ventilation modes.

Conclusions Measuring PFR is safe after cardiac surgery in the ICU and may constitute a novel method of continuously monitoring the small airways in real time. A low PFR during mechanical ventilation may correlate to a gentle ventilation strategy. PFR increases as the patient transitions from controlled mechanical ventilation to autonomous breathing, which most likely occurs as recruitment by the diaphragm opens up more distal airways. Different ventilation modes resulted in unique particle patterns and could be used as a fingerprint for the different ventilation modes.

\section{Introduction}

Ventilator-induced lung injury (VILI) can be an adverse consequence of mechanical ventilation [1-4]. Adopting ventilator strategies which reduce VILI is an important goal in ventilatory management. In acute respiratory distress syndrome (ARDS), lung protective ventilation with low tidal volumes and limited airway pressure has significantly reduced mortality among those patients [1, 5]. Among the different ventilation modes, pressure-controlled ventilation (PCV) is often suggested to reduce the risk of developing VILI due to the potential ramifications of high pressure when compared to the use of volume-controlled ventilation (VCV) [6, 7]. However, in meta-analysis studies, there is yet to be a definitive conclusion regarding the advantage of either PCV or VCV [4]. The benefits relative to the potential damage incited by recruitment manoeuvres is also an area of continuous debate especially in the most vulnerable groups of 
patients, such as those with ARDS. To this day, the evidence for the benefits without causing any additional harm are not conclusive $[8,9]$. There is still ongoing debate surrounding the optimal recruitment manoeuvre to use in both methods of ventilation and at what time during the course of mechanical ventilation the recruitment manoeuvre should be performed [10,11]. Patients with severe ARDS, who are responders to recruitment manoeuvre, should be treated according to the open lung concept with low tidal volumes and high positive end expiratory pressure (PEEP) after a recruitment manoeuvre. Conversely, patients who are not responders should be treated with reduced PEEP without any recruitment manoeuvres to avoid overdistension of alveoli [1]. However, despite pronounced ventilation strategies, VILI still occurs, indicating that clinicians need new or improved diagnostic tools to monitor mechanical ventilation in avoidance of VILI.

Exhaled breath particles (EBPs) are thought to originate from the respiratory tract lining fluid (RTLF) that covers the epithelial surface of the distal parts of the lung [12-15]. EBPs are transmitted in exhaled air during the opening and closing of small airways and can be measured as particle flow rate (PFR) using a PExA machine. The identity of particles collected using the PExA technique has been shown to be consistent with the particles collected from bronchoalveolar lavage fluid, indicating that EBPs originate from the RTLF [15]. In a recent publication, increased PFR was strongly connected to lung injury [16]. The PFR significantly increased indicating lung injury prior to other clinical indicators of injury, including respiratory parameters and the cytokine response in the blood. The implication is that PFR could be used as an early signal of lung injury [16].

The present study was designed to explore if and how PFR could be used to monitor mechanical ventilation in different ventilation modes. We further explored if different ventilator settings resulted in different PFR patterns in post-operative patients on mechanical ventilation without lung pathology. Furthermore, the effect of recruitment manoeuvre during different ventilation modes was investigated.

Method

A prospective randomised controlled clinical study was performed in patients on mechanical ventilation at Lund University Hospital in Sweden during 2019. The study was approved by the local ethics committee, EPN Dnr 2018/129. The study consisted of 30 patients (22 males, 8 females) undergoing elective open-heart surgery: coronary artery bypass grafting (CABG) $(n=15)$, aortic valve replacement surgery (AVR) $(n=10)$ or a combination of CABG and AVR $(n=5)$. The mean age was 69 years, ranging from 44 to 83. All patients enrolled in the study were presented with information regarding the study and signed an informed consent form.

All patients were initially sedated using Propofol-®Lipuro (B Braun Medical AB, Danderyd, Sweden) $20 \mathrm{mg} \cdot \mathrm{mL}^{-1}$ at an infusion rate of $2-3 \mathrm{mg} \cdot \mathrm{kg}^{-1} \cdot \mathrm{h}^{-1}$ during the time in the intensive care unit (ICU). Norepinephrine (Pfizer AB, Sollentuna, Sweden) $40 \mu \mathrm{g} \cdot \mathrm{mL}^{-1}$, at infusion rates between 0.05 and $2 \mu \mathrm{g} \cdot \mathrm{kg}^{-1} \cdot \mathrm{min}^{-1}$, was used to maintain mean arterial pressure (MAP) above $65 \mathrm{mmHg}$. MAPs above accepted levels were treated with Nitroglycerin Abcur (Abcur AB, Helsingborg, Sweden) $1 \mathrm{mg} \cdot \mathrm{mL}^{-1}$, at infusion rates between 0.25 and $5 \mu \mathrm{g} \cdot \mathrm{kg}^{-1} \cdot \mathrm{min}^{-1}$. In the case of post-operative pain and shivering, intravenous oxycodone $10 \mathrm{mg} \cdot \mathrm{mL}^{-1}$ (Mundipharma $\mathrm{AB}$, Göteborg, Sweden), Petidine $50 \mathrm{mg} \cdot \mathrm{mL}^{-1}$ (Meda $\mathrm{AB}$, Solna, Sweden) or clonidine $150 \mu \mathrm{g} \cdot \mathrm{mL}^{-1}$ (Boehringer Ingelheim AB, Stockholm, Sweden) were administered.

\section{Inclusion criteria}

Patients included in the study were those with normal lung function undergoing elective open-heart surgery who would receive post-operative treatment with mechanical ventilation at the cardiothoracic ICU.

\section{Exclusion criteria}

Patients were excluded if they had COPD stage 1-4, pulmonary fibrosis or other kinds of lung pathology. They were also excluded if they were currently a smoker or a recent smoker ( $<6$ months). Other exclusion criteria included an ejection fraction $<45 \%$, chronic renal failure or chronic liver failure.

\section{PEXA device and measurements}

A customised PExA 2.0 device (PExA, Gothenburg, Sweden) was connected on the expiratory limb of the respiratory circuit. A mouthpiece containing a one-way valve was used to prevent mixture of inspiratory and expiratory air. PFR was measured continuously from the airways by the PExA device for the entire period during which the patient was intubated in the ICU. The PExA device conducts measurements using an optical particle counter (OPC) and by impaction on a filter. It can thereby analyse exhaled particle flow and exhaled particle size distribution, as described in detail in previous studies [17, 18]. The PExA device 


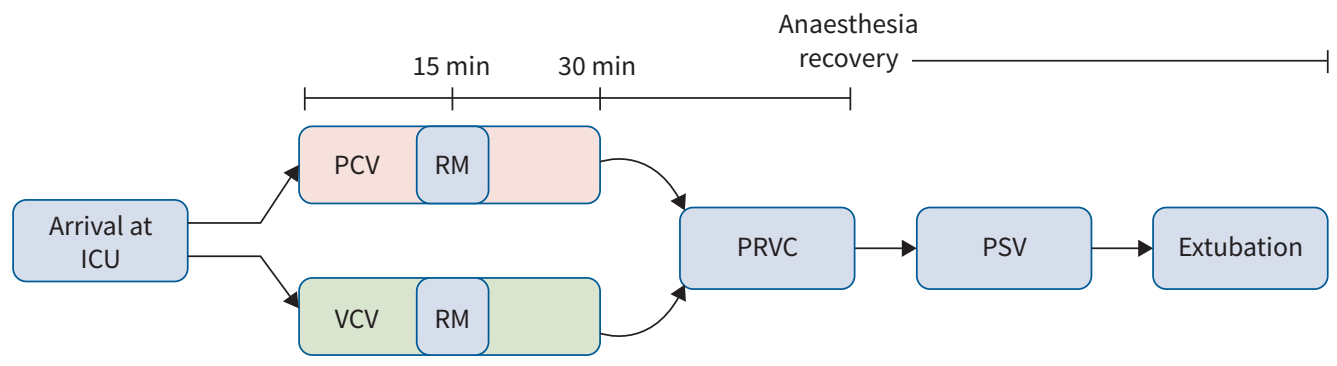

FIGURE 1 Flowchart after arrival at the intensive care unit (ICU). The patients were randomised to either volume-controlled ventilation (VCV) or pressure-controlled ventilation (PCV) including a recruitment manoeuvre (RM) as the first line of treatment. The mechanical ventilation was thereafter set to pressure-regulated volume control (PRVC) in all patients until recovery from anaesthesia when the ventilator was switched to pressure-support ventilation (PSV) to facilitate spontaneous breathing. Figure created with BioRender.com.

measures particles in the size range $0.41 \mu \mathrm{m}$ to $4.55 \mu \mathrm{m}$ by the OPC and divides them into eight different size groups, as follows: particle 1: $0.48(0.41-0.55) \mu \mathrm{m}$; particle 2: $0.59(0.55-0.70) \mu \mathrm{m}$; particle $3: 0.75$ $(0.70-0.92) \mu \mathrm{m}$; particle 4: $0.98(0.92-1.14) \mu \mathrm{m}$; particle 5: $1.22(1.14-1.44) \mu \mathrm{m}$; particle $6: 1.67(1.44-$ 2.36) $\mu \mathrm{m}$; particle 7: $2.52(2.36-2.98) \mu \mathrm{m}$; particle 8: 3.37 (2.98-4.55) $\mu \mathrm{m}$ [17].

A study timeline is shown in figure 1. Upon arrival into the ICU, patients were randomised to either VCV or PCV during the first $30 \mathrm{~min}$ after arrival at the ICU including a recruitment manoeuvre $15 \mathrm{~min}$ after initiation of ventilation. The recruitment manoeuvre was performed over 1 min using a PEEP of $10 \mathrm{cmH}_{2} \mathrm{O}$, a respiratory rate of 4 breaths $\cdot \mathrm{min}^{-1}$ and an inspiratory-expiratory ratio (I:E) of 2:1. All other settings were kept unchanged during the recruitment manoeuvre. A representative measurement of 3 min before and $3 \mathrm{~min}$ after the recruitment manoeuvre was chosen for statistical analysis of PFR during the recruitment manoeuvre. PFR was measured during VCV and PCV at two time periods, before and after recruitment manoeuvre to obtain a representative value.

When the patient awoke from anaesthesia, the ventilator mode was set to PSV to allow spontaneous breathing. Train-of-four (TOF) was analysed in close proximity to the recruitment manoeuvre to ensure that the muscle relaxation drugs (rocuronium bromide) given during surgery did not have any impact on the measurements.

Vital parameters and ventilatory settings

Vital parameters were measured, in addition to the standard monitoring at the ICU; at arrival, before recruitment manoeuvre, after recruitment manoeuvre and before extubation. Measurements are presented in table 1 . The ventilator settings at arrival were individual according to local guidelines (tidal volume $\left(\mathrm{V}_{\mathrm{t}}\right)$ of 6-8 $\mathrm{mL} \cdot \mathrm{kg}^{-1}$, PEEP of $5 \mathrm{cmH}_{2} \mathrm{O}$, inspiratory oxygen fraction $\left(F_{\mathrm{IO}_{2}}\right)$ of $30-50 \%$, peak inspiratory pressure (PIP) $<25 \mathrm{cmH}_{2} \mathrm{O}$ and end tidal $\mathrm{CO}_{2}$ between 34.5 and $45 \mathrm{mmHg}$ ); respiratory parameters during measurements are presented in table 2.

\section{Statistics}

A power analysis was performed prior to the study. Descriptive statistics with mean and standard error of the mean (SEM) were used in results and figures. GraphPad Prism 8 was used for statistical analysis. MannWhitney test and Kruskal-Wallis test were performed (depending on data distribution) to analyse if statistically significant difference was achieved among particles in exhaled air. Statistical significance was defined as $\mathrm{p}<0.0001\left(^{* * * *}\right), \mathrm{p}<0.001\left(^{* * *}\right), \mathrm{p}<0.01(* *), \mathrm{p}<0.05\left(^{*}\right)$ and $\mathrm{p}>0.05$ (not significant).

Results

Of the 36 patients assessed for eligibility, six did not meet the inclusion criteria and were therefore excluded from the study. The remaining 30 patients were subsequently randomised to either VCV or PCV during the first $30 \mathrm{~min}$ upon arrival at the ICU. After this thirty-minute period, which included a recruitment manoeuvre at $15 \mathrm{~min}$, patients were placed on pressure regulated volume control (PRVC) until they have gained consciousness and are then placed on pressure support ventilation (PSV) until extubation. All randomised patients received allocated interventions. No patients were lost to follow-up. Patient demographics are shown in table 3. 
TABLE 1 Haemodynamic parameters, train-of-four (TOF) and blood gases during measurement at arrival in the intensive care unit (baseline), before and after recruitment manoeuvre, and before extubation

\begin{tabular}{|c|c|c|c|c|}
\hline Parameters & Baseline & Before recruitment manoeuvre & After recruitment manoeuvre & Ending \\
\hline $\mathrm{SBP} \mathrm{mmHg}$ & $104.9 \pm 3.1$ & $116.4 \pm 3.1$ & $118.2 \pm 2.4$ & $127.6 \pm 3.5$ \\
\hline $\mathrm{DBP} \mathrm{mmHg}$ & $54.5 \pm 1.8$ & $60.2 \pm 2.2$ & $60.9 \pm 2.0$ & $62.7 \pm 2.1$ \\
\hline MAP $\mathrm{mmHg}$ & $69.8 \pm 2.1$ & $77.3 \pm 2.3$ & $77.7 \pm 2.0$ & $81.2 \pm 2.2$ \\
\hline CVP $\mathrm{mmHg}$ & $1.3 \pm 0.8$ & $3.4 \pm 0.7$ & $3.4 \pm 0.8$ & $6.6 \pm 0.9$ \\
\hline HR beats $\cdot \min ^{-1}$ & $76.9 \pm 1.9$ & $75.8 \pm 1.9$ & $74.1 \pm 2.2$ & $80.4 \pm 2.2$ \\
\hline SAT $\%$ & $98.5 \pm 0.4$ & $98.8 \pm 0.4$ & $99.5 \pm 0.2$ & $97.8 \pm 0.4$ \\
\hline TOF $\%$ & $92.9 \pm 5.3$ & & & \\
\hline $\mathrm{PH}$ & $7.38 \pm 0$ & & & $7.35 \pm 0$ \\
\hline$P_{\mathrm{CO}_{2}} \mathrm{mmHg}$ & $38.7 \pm 0.8$ & & & $43.2 \pm 1.1$ \\
\hline$P_{\mathrm{O}_{2}} \mathrm{mmHg}$ & $132.8 \pm 5.7$ & & & $98.3 \pm 3.3$ \\
\hline
\end{tabular}

Ventilation with PRVC resulted in the lowest PFR

When comparing different ventilation modes, PRVC produced the lowest PFR at a rate of 77.2 \pm 34.4 particles $\cdot \mathrm{min}^{-1}$ compared to VCV 83.7 $\pm 16.8(\mathrm{p}=0.0285)$ and PCV 439.1 $\pm 153(\mathrm{p}=0.0149)$. However, there were no significant differences between the VCV and PCV group $(p=0.3980)$. The results are shown as mean \pm SEM (figure 2).

Ventilation with PSV resulted in significantly higher PFR compared to all other ventilation modes used When the patient awoke from anaesthesia, the ventilator mode was set to PSV to allow spontaneous breathing. The PFR significantly increased during PSV compared to all other ventilation modes studied.

The PFR during PSV was $2249 \pm 426$ particles $\cdot \mathrm{min}^{-1}$ compared to PFR during PRVC $77.2 \pm 43.4$ particles $\cdot \min ^{-1}(\mathrm{p}<0.0001)$ (figure 2$)$.

Recruitment manoeuvre did not result in increased PFR

A recruitment manoeuvre was conducted in patients in both VCV and PCV mode but did not result in any significant changes in PFR before or after the manoeuvre. For example, the PFR in VCV before the recruitment manoeuvre was $77.6 \pm 19.3$ particles $\cdot \mathrm{min}^{-1}$ and after the recruitment manoeuvre was $89.8 \pm 28$ particles $\cdot \min ^{-1}(\mathrm{p}=0.6529)$ (figure 3$)$.

TABLE 2 Ventilator settings and respiratory parameters at arrival in the intensive care unit (baseline), before and after recruitment manoeuvre, and before extubation

\begin{tabular}{|c|c|c|c|c|}
\hline Parameters & Baseline & Before recruitment manoeuvre & After recruitment manoeuvre & Ending \\
\hline Ventilation mode & VCV:PCV (1:1) & VCV:PCV (1:1) & PRVC & PSV \\
\hline PEEP $\mathrm{CmH}_{2} \mathrm{O}$ & $5.2 \pm 0.1$ & $5.2 \pm 0.1$ & $5.1 \pm 0.1$ & $2.4 \pm 0.2$ \\
\hline PIP $\mathrm{cmH}_{2} \mathrm{O}$ & $17.0 \pm 0.9$ & $17.0 \pm 0.5$ & $14.6 \pm 0.4$ & $12.8 \pm 0.2$ \\
\hline$P_{\text {mean }} \mathrm{CmH}_{2} \mathrm{O}$ & $8.9 \pm 0.2$ & $9.0 \pm 0.3$ & $8.7 \pm 0.2$ & $5.1 \pm 0.2$ \\
\hline PS $\mathrm{cmH}_{2} \mathrm{O}$, in PCV and PSV & $11.0 \pm 0.6$ & $10.9 \pm 0.6$ & & $10.0 \pm 0.1$ \\
\hline RR breaths $\cdot \mathrm{min}^{-1}$ & $14.4 \pm 0.2$ & $14.4 \pm 0.3$ & $14.3 \pm 0.2$ & $16.2 \pm 1.0$ \\
\hline$V_{\mathrm{T}}$ INSP mL & $509.0 \pm 15.6$ & $518.5 \pm 16.2$ & $506.6 \pm 11.2$ & $586.5 \pm 37.8$ \\
\hline$V_{\mathrm{T}}$ EXP mL & $501.7 \pm 15.8$ & $507.0 \pm 15.9$ & $496.1 \pm 10.7$ & $580.7 \pm 41.5$ \\
\hline$M V L \cdot \min ^{-1}$ & $7.3 \pm 0.3$ & $7.2 \pm 0.2$ & $7.1 \pm 0.2$ & $8.6 \pm 0.3$ \\
\hline$C_{\text {dyn }} \mathrm{mL} / \mathrm{cmH}_{2} \mathrm{O}$ & $51.5 \pm 2.8$ & $53.3 \pm 2.7$ & $54.4 \pm 2.0$ & $52.8 \pm 4.4$ \\
\hline $\mathrm{F}_{\mathrm{IO}_{2}} \%$ & $42.0 \pm 1.0$ & $40.7 \pm 0.8$ & $40.0 \pm 0.9$ & $32.2 \pm 0.6$ \\
\hline $\mathrm{I}: \mathrm{E}^{2}$ & $1: 2$ & $1: 2$ & $1: 2$ & \\
\hline
\end{tabular}

Data presented as mean \pm SEM. PEEP: positive end expiratory pressure; PIP: peak inspiratory pressure; $P_{\text {mean }}$ : mean pressure; PS: pressure support; RR: respiratory rate; $V_{\mathrm{T}}$ : tidal volume; $\mathrm{MV}$ : minute ventilation; $C_{\mathrm{dyn}}$ : dynamic compliance; $F_{\mathrm{IO}_{2}}$ : inspiratory oxygen fraction; I:E: inspiratory-expiratory ratio; VCV: volume-controlled ventilation; PCV: pressure-controlled ventilation; PRVC: pressure-regulated volume control; PSV: pressure support ventilation. 


\begin{tabular}{|c|c|c|c|}
\hline & $\begin{array}{l}\text { Volume-controlled } \\
\text { ventilation (VCV) }\end{array}$ & $\begin{array}{l}\text { Pressure-controlled } \\
\text { ventilation (PCV) }\end{array}$ & Total \\
\hline Patients, $\mathrm{n}$ & 15 & 15 & 30 \\
\hline Sex male/female, $\mathrm{n}(\%)$ & $13(87) / 2(13)$ & $9(60) / 6(40)$ & $22(73) / 8(27)$ \\
\hline \multicolumn{4}{|l|}{ Type of surgery, $n$} \\
\hline CABG & 8 & 7 & 15 \\
\hline AVR & 6 & 4 & 10 \\
\hline CABG and AVR & 1 & 4 & 5 \\
\hline Age years, mean \pm sD & $70.3 \pm 11.1$ & $67.5 \pm 9.4$ & $68.9 \pm 10.2$ \\
\hline Smoking & No & No & No \\
\hline Normal chest radiograph & Yes & Yes & Yes \\
\hline Normal renal function & Yes & Yes & Yes \\
\hline$E F>55 \%$ & Yes & Yes & Yes \\
\hline
\end{tabular}

Distribution of particles according to size did reflect significant differences between the different ventilation modes implying that the PEXA device could be used as a fingerprint diagnostic in mechanical ventilation

The PExA device divides particles according to particle impaction and can thereby analyse size by distribution into eight different size groups, described in detail in previous studies $[17,18]$. When studying the particle size distribution between the ventilation modes, we discovered a significantly higher number of particles seen in PSV for all particle size groups $(1-8)$ compared to the other ventilation modes $(\mathrm{p}<0.0001)$ (figure 4).

When analysing particle size separately we found a significantly lower PFR in particle size groups 1-4 comparing PRVC to PCV and also a significantly lower PFR in particle size groups 1-3 comparing PRVC to VCV. No difference was found for particle size groups 5-8 (figure 4).

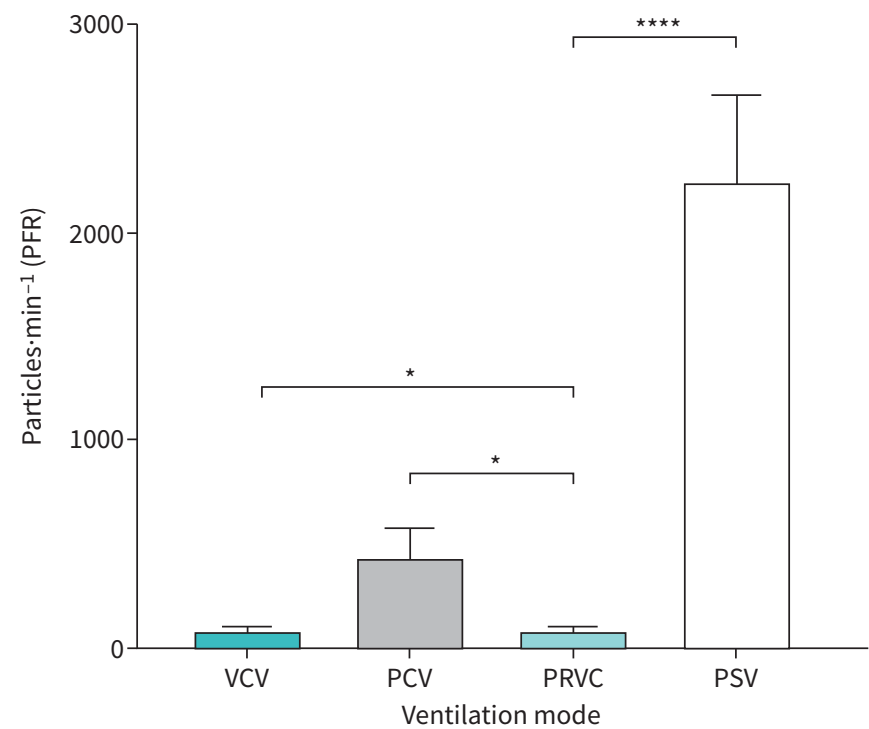

FIGURE 2 There was a significantly lower particle flow rate (PFR) when using pressure-regulated volume control (PRVC) compared to volume-controlled ventilation (VCV) and pressure-controlled ventilation (PCV). Ventilation with pressure support ventilation (PSV) resulted in significantly higher PFR compared to PRVC. Statistical tests were conducted using the Mann-Whitney test. Statistical significance was defined as $p<0.0001$ $\left(^{\star \star \star \star}\right)$ and $p<0.05\left(^{\star}\right)$. 


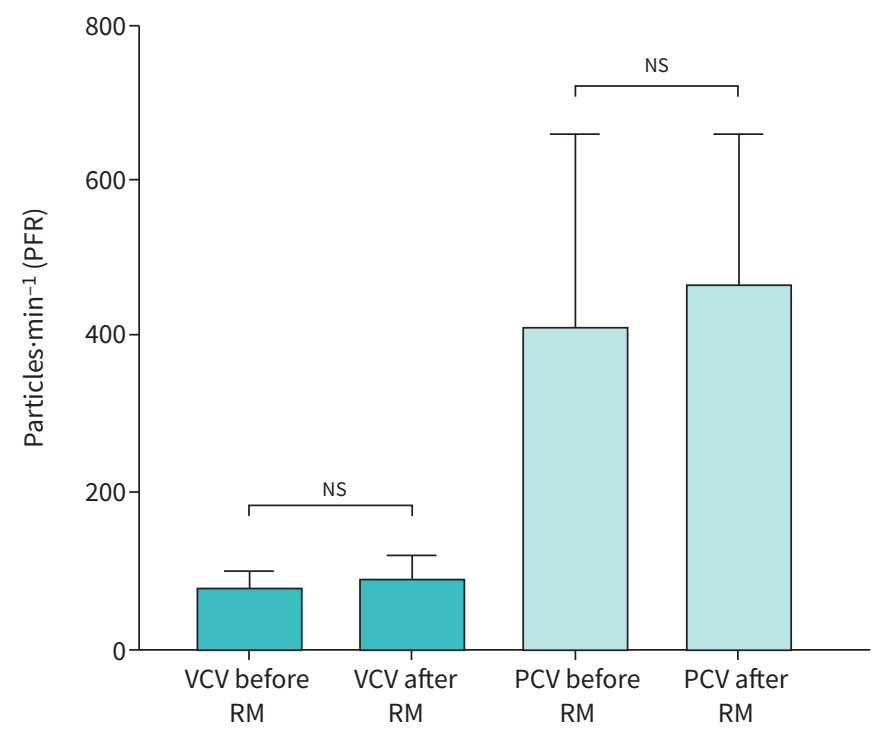

FIGURE 3 No significant difference was found in either ventilator mode when comparing particle flow rate (PFR) before and after the recruitment manoeuvre (RM). There was no significant difference between volume-controlled ventilation (VCV) and pressure-controlled ventilation (PCV) before and after the recruitment manoeuvre. Ns: not significant.

\section{Discussion}

Conventional monitoring of mechanical ventilation in the ICU is unable to detect subtle changes or stressors in the lung which precede the onset of lung injury. New techniques - and ideally those which are noninvasive - should strive to detect early changes in the lung with the goal of optimising mechanical ventilation. Such strategies could be personalised for the patient to reduce VILI. Considering that one contributing factor to VILI may be the repeated opening and closing of the distal airways during mechanical ventilation [2], a method of monitoring the activity of these airways would be clinically useful. The PFR as measured by the PExA device reflects the EBPs transmitted by these small airways and thus, a low PFR is theorised to correlate with reduced opening and closure of the airways and consequently gentler ventilation.

It is proposed that PFR in the clinic is a tool to measure changing conditions which a clinician could then utilise to gain a clearer picture of the clinical scenario. For example, increasing or decreasing PFR could be secondary to a change in ventilatory settings, which would be readily apparent to a clinician, or could be the result of patient transitioning from mechanical breathing to spontaneous breathing, or lung pathology. The clinician could use PFR as a tool to monitor lung status, and thus, the relationship of PFR to ventilation mode was examined here. In this study, changes in ventilation mode and comparison between modes was correlated to changes in PFR.

A previous clinical study on lung transplant patients showed no difference in PFR using the ventilation modes VCV and PCV [19]. This same observation was reproduced in the present study. According to clinical guidelines, PRVC is considered a gentle ventilation mode when a specific volume is desired, as it also maintains the lowest possible pressure. A previous study compared PRVC to VCV and found significantly lower PIP when ventilating with PRVC [20]. In the present study, a significantly lower PFR was observed when using PRVC relative to both PCV and VCV.

Additionally, this study found that PSV as a mode resulted in a higher PFR compared to all other modes. In theorising the cause of such higher rates, the mechanics of breathing should be considered. While the patient is sedated, both the diaphragm and the accessory muscles are affected by sedatives relative to conscious breathing. Post-operatively, as sedation wears off and the ventilation mode is transitioned to PSV, the patient takes over increasingly more of the respiratory work. In this transition from controlled mechanical breathing to more conscious control by the patient with support from the ventilator, there is a significant increase in PFR. This increase is hypothesised to be attributed to the relative work of increasingly active respiratory muscles as active breathing seems to open previously closed alveoli. This reiterates the result of higher PFR in spontaneously breathing patients relative to ventilated patients, as previously published [19]. 

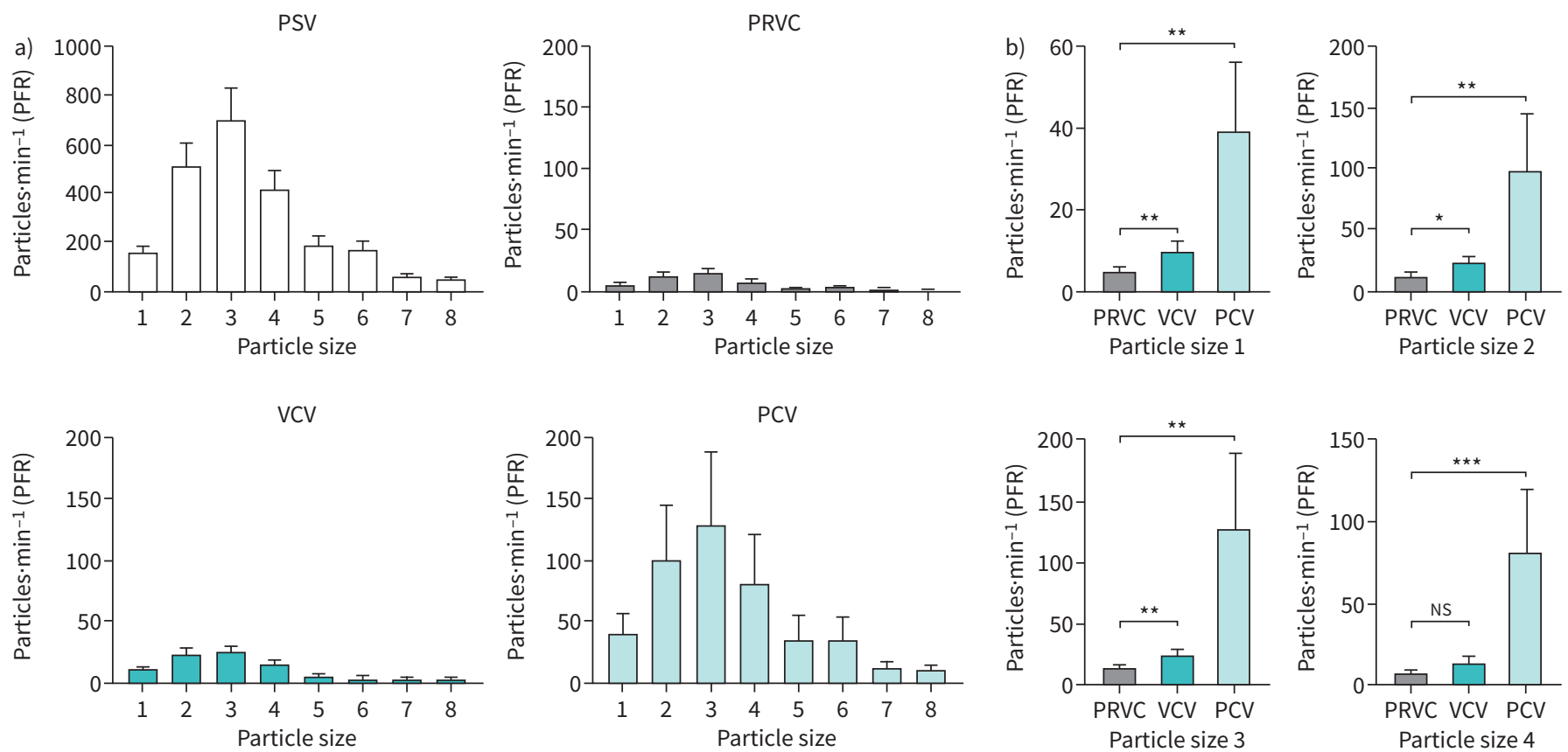

FIGURE 4 a) Distribution of particles according to size in the different ventilation modes. When looking at particle flow rate (PFR) according to the different particle size bins (1-8) we can distinguish distinct patterns linked to different ventilation modes. In pressure support ventilation (PSV), there was a significantly higher PFR for all particle sizes (1-8) compared to the other ventilation modes. Statistical tests were conducted using the Kruskal-Wallis test. b) PFR for particle sizes 1-4 in the different ventilation modes. A significant difference was found between pressure-regulated volume control (PRVC) and pressure-controlled ventilation (PCV) for particle sizes 1-4. A significant difference between PRVC and volume-controlled ventilation (VCV) was found for particle sizes 1-3. No difference was found when comparing VCV to PCV for particle sizes 1-4. Statistical significance was defined as $p<0.001\left(^{\star \star \star}\right), p<0.01\left(^{\star \star}\right), p<0.05\left(^{\star}\right)$ and $p>0.05$ (not significant, Ns).

A recent study has demonstrated an increase in PFR preceding clinical signs of ARDS in a large animal model [16], and another study in lung transplanted patients has additionally shown higher PFR as a clinical indicator of primary graft dysfunction, a type of acute lung injury [19]. This would appear to indicate that particles in exhaled breath increase with pathology of the lung and resultantly may be an early indicator of damage to the lung. None of the patients in the present study showed signs of increased PFR during the time of the study, and there were no other clinical signs of deteriorating condition, and thus neither PCV, VCV or PRVC ventilation modes used under sedation appeared to be harmful to the lungs in patients with normal lung function. We have noted that there is inter-individual variation in PFR values, which could be integrated into the assessment of each individual patient. Further studies could include a longitudinal observation following an individual's change in PFR over longer surgical procedures or longer ICU stays where a patient may be on mechanical ventilation for a prolonged period.

The PExA device measures particles in the size range of $0.41 \mu \mathrm{m}$ to $4.55 \mu \mathrm{m}$, which are then divided into eight different size groups by the OPC [17]. The distribution regarding particle count between the bin sizes 1-8 differed between the different ventilation modes in the present study. A significantly higher number of particles was seen using PSV for all particle size groups, compared to the other ventilation modes studied. When comparing PRVC, VCV and PCV, significant differences were seen between bin sizes 1-4, which established different particle patterns in these different modes. This could imply that particle patterns have the potential to act as a fingerprint of distinct change or pathology. Further study will include the use of PFR in different lung pathologies to establish if the phenomena of distinctive patterns, as noted in this study, holds true for disease states. Characterisation of the composition of EBPs during different ventilation modes and clinical circumstances could provide new insight into physiology and pathology of the lung.

Our hypothesis regarding high PFR during PSV ventilation follows that while initiating their own breath in this mode, patients use a breathing pattern similar to that of spontaneously breathing patients. As seen in a previous study, PFR in spontaneously breathing patients is significantly higher than that found in patients placed on mechanical ventilation [21]. The use of the diaphragm, as in spontaneous breathing, promotes the opening and closing of the distal airways and may thus influence the production of EBPs [12]. While 
under PRVC ventilation, the machine uses positive pressure to administer air. In PSV ventilation, however, the patient initiates breathing by generating negative pressure using the diaphragm and respiratory muscles. The ventilator then supports the patient's efforts by using positive pressure to ensure each breath constitutes complete tidal breathing. PEEP is applied in any ventilatory mode as it prevents the collapse of the small distal airways.

The recruitment manoeuvre implemented in this study has been previously published in both a preclinical porcine study of abdominal surgery and in a clinical study of lung transplant patients. These publications showed a significantly higher PFR after the recruitment manoeuvre when using VCV [18, 19]. This provided the rationale for using the same recruitment manoeuvre here, but we did not see any differences in PFR between the different ventilation modes within this patient population. This result should be considered in the light of the use of patients without lung pathology. In the study conducted by BROBERG et al. [19], patients had undergone lung transplantation. Lung transplant recipients experience pulmonary inflammatory reactions post-operatively and tissue trauma in the lungs from the surgery itself which might explain the response to the recruitment manoeuvre as seen by the PFR. When considering the preclinical study on PFR following recruitment manoeuvres in a porcine model, there are several potential explanations as to why the results of that study differ: anatomic differences could be considered and the length of time on mechanical ventilation [18]. There might be several explanations as to why we did not observe a higher PFR after recruitment manoeuvre in the present study. Before leaving the operating room, all patients underwent a recruitment manoeuvre according to local clinical practice. The measurements for the present study started in the ICU $\sim 1 \mathrm{~h}$ after leaving the operating room.

In considering the results and conclusions which can be drawn from the present study, we must also take note of the limitations. The scope of this study was focused on the analysis of particle rate and patterns of distribution, as this research aimed to demonstrate the utility of PFR measurement as a bedside clinical tool. As particles were collected onto a membrane, future analysis could include investigation of specific biomarkers to develop a more mechanistic view of EBPs in pathological conditions. Additionally, the recruitment manoeuvre used in the ICU was noted to be a gentler manoeuvre compared to that in the operating room. As the patients have already undergone a more vigorous recruitment manoeuvre in the operating room while surgeons can monitor an open chest, the recruitment manoeuvre used in the ICU is gentler in light of the risk of bleeding and possible rupture of anastomoses in the immediate post-operative phase. Future study of PFR in relation to recruitment manoeuvres would need to be conducted in a different patient population, as we would not subject the patients in this study to a recruitment manoeuvre more aggressive than that which we implemented. Furthermore, as previously discussed, the patients included here are those who underwent routine cardiac surgery and therefore had briefer stays in the ICU. Resultantly, the time spent on ventilation was relatively short. In future, we would consider including a broader spectrum of patients with lung and other types of pathology who stay in the ICU longer. This could be achieved by extending the study to other centres through collaboration. This would allow us to both study a less homogeneous population as well as conduct analyses of PFR over a greater period of time.

This study reiterates the finding that measurement of PFR using the PExA device is safe in conjunction with mechanical ventilation $[18,19,21]$. This was concluded as there were no signs of significant air leakage, haemodynamic impact or increased peak pressures which could be harmful to the patients.

\section{Conclusions}

PFR can be used to monitor mechanical ventilation with the future aim of personalising ventilator settings to each individual. The results suggest further research should investigate PRVC relative to VCV and PCV as the lower PFR could indicate that this is a gentler method of ventilation in a fully sedated patient where the goal is to avoid mechanical stress on the lung. Furthermore, the various ventilator settings explored in this study resulted in different distributions of exhaled particles binned by size. This could serve as the basis for studying PFR in terms of distinctive distribution patterns as a fingerprint of lung and ventilatory condition. We further concluded that a higher PFR was measured when ventilating in PSV mode compared to PRVC, which may reflect the added contribution of the work of breathing initiated by the patient. The findings of this study support the further investigation of PFR in terms of both rate and particle distribution as a promising clinical tool by which to assess the contribution of ventilatory mode to patient care.

Acknowledgements: The authors thank all co-workers at the cardiothoracic intensive care unit at Lund University Hospital.

This study is registered at www.ClinicalTrials.gov with identifier number NCT04503057. Data availability: Individual deidentified participant data (including data dictionaries) will be shared including the study protocol and 
statistical analysis plan. The data will become available at the time of publication with no limitation in length. The data will be accessible to researchers whose proposed use of the data has been approved by an independent reviewer committee used for this purpose. Please contact author for data requests.

Author contributions: S. Lindstedt, S. Hyllén, M. Malmsjö, E. Broberg, M. Stenlo and F. Hallgren participated in the design of the study. S. Lindstedt wrote the application for the ethical approval. F. Hallgren, M. Stenlo, S. Hyllén and E. Broberg performed the PExA measurements in the intubated patients. S. Linsdtedt, M. Stenlo and F. Hallgren analysed the study results. S. Lindstedt, M. Stenlo, A. Niroomand and F. Hallgren drafted the manuscript. All authors read and approved the final manuscript.

Conflict of interest: All authors declare that they have no competing interests.

Support statement: This work was funded by the Wallenberg Molecular Medicine Fellowship, the Knut and Alice Wallenberg Foundation, the Marcus and Marianne Wallenberg Foundation and the ALF foundation. Funding information for this article has been deposited with the Crossref Funder Registry.

\section{References}

1 van der Zee P, Gommers D. Recruitment maneuvers and higher PEEP, the so-called open lung concept, in patients with ARDS. Crit Care 2019; 23: 73.

2 Beitler JR, Malhotra A, Thompson BT. Ventilator-induced lung injury. Clin Chest Med 2016; 37: 633-646.

3 Fan E, Brodie D, Slutsky AS. Acute respiratory distress syndrome: advances in diagnosis and treatment. JAMA 2018; 319: 698-710

4 Chacko B, Peter JV, Tharyan P, et al. Pressure-controlled versus volume-controlled ventilation for acute respiratory failure due to acute lung injury (ALI) or acute respiratory distress syndrome (ARDS). Cochrane Database Syst Rev 2015; 1: Cd008807.

5 Needham DM, Colantuoni E, Mendez-Tellez PA, et al. Lung protective mechanical ventilation and two year survival in patients with acute lung injury: prospective cohort study. BMJ 2012; 344: e2124.

6 Gao S, Zhang Z, Brunelli A, et al. The Society for Translational Medicine: clinical practice guidelines for mechanical ventilation management for patients undergoing lobectomy. J Thorac Dis 2017; 9: 3246-3254.

7 Tan J, Song Z, Bian Q, et al. Effects of volume-controlled ventilation vs. pressure-controlled ventilation on respiratory function and inflammatory factors in patients undergoing video-assisted thoracoscopic radical resection of pulmonary carcinoma. J Thorac Dis 2018; 10: 1483-1489.

8 Dyhr $\mathrm{T}$, Nygard $\mathrm{E}$, Laursen $\mathrm{N}$, et al. Both lung recruitment maneuver and PEEP are needed to increase oxygenation and lung volume after cardiac surgery. Acta Anaesthesiol Scand 2004; 48: 187-197.

9 Futier E, Constantin JM, Petit A, et al. Positive end-expiratory pressure improves end-expiratory lung volume but not oxygenation after induction of anaesthesia. Eur J Anaesthesiol 2010; 27: 508-513.

10 Cui $\mathrm{Y}, \mathrm{Cao} \mathrm{R}, \mathrm{Li} \mathrm{G}$, et al. The effect of lung recruitment maneuvers on post-operative pulmonary complications for patients undergoing general anesthesia: a meta-analysis. PLOS ONE 2019; 14: e0217405.

11 Cui Y, Cao R, Wang Y, et al. Lung recruitment maneuvers for ARDS patients: a systematic review and meta-analysis. Respiration 2020: 99: 264-1276.

12 Bake B, Larsson P, Ljungkvist G, et al. Exhaled particles and small airways. Respir Res 2019; 20: 8.

13 Almstrand AC, Bake B, Ljungstrom E, et al. Effect of airway opening on production of exhaled particles. J Appl Physiol (1985) 2010; 108: 584-588.

14 Almstrand AC, Ljungstrom E, Lausmaa J, et al. Airway monitoring by collection and mass spectrometric analysis of exhaled particles. Anal Chem 2009; 81: 662-668.

15 Bredberg A, Gobom J, Almstrand AC, et al. Exhaled endogenous particles contain lung proteins. Clin Chem 2012; 58: 431-440.

16 Stenlo M, Hyllen S, Silva IAN, et al. Increased particle flow rate from airways precedes clinical signs of ARDS in a porcine model of LPS-induced acute lung injury. Am J Physiol Lung Cell Mol Physiol 2020; 318: L510-L517.

17 Broberg E, Wlosinska M, Algotsson L, et al. A new way of monitoring mechanical ventilation by measurement of particle flow from the airways using Pexa method in vivo and during ex vivo lung perfusion in DCD lung transplantation. Intensive Care Med Exp 2018; 6: 18.

18 Broberg E, Pierre L, Fakhro M, et al. Different particle flow patterns from the airways after recruitment manoeuvres using volume-controlled or pressure-controlled ventilation. Intensive Care Med Exp 2019; 7: 16.

19 Broberg E, Hyllen S, Algotsson L, et al. Particle flow profiles from the airways measured by PExA differ in lung transplant recipients who develop primary graft dysfunction. Exp Clin Transplant 2019; 17: 803-812.

20 Guldager H, Nielsen SL, Carl P, et al. A comparison of volume control and pressure-regulated volume control ventilation in acute respiratory failure. Crit Care 1997; 1: 75-77.

21 Broberg E, Andreasson J, Fakhro M, et al. Mechanically ventilated patients exhibit decreased particle flow in exhaled breath as compared to normal breathing patients. ERJ Open Res 2020; 6: 00198-2019. 